\title{
Cooperative Spectrum Sensing in Cognitive Radio Networks
}

\author{
Ghurumuruhan Ganesan†and Ye (Geoffrey) Li \\ School of Electrical and Computer Engineering \\ Georgia Institute of Technology, Atlanta, Georgia 30332-0250
}

\begin{abstract}
In cognitive networks, cognitive (unlicensed) users need to continuously monitor spectrum to detect the presence of primary (licensed) users. In this paper, we illustrate the benefits of cooperation in cognitive radio. We show that by allowing the cognitive radios operating in the same band to cooperate we can reduce the detection time and thus increasing their agility. We first consider the case of two cognitive users and show how the inherent asymmetry in the network can be exploited to increase the probability of detection. We then extend our work to multiple cognitive user networks. We also propose a practical algorithm which allows cooperation in random networks.
\end{abstract}

Index Terms - cognitive radio, cooperative diversity, agility gain, detection time.

\section{INTRODUCTION}

Recently there has been tremendous interest in the field of software defined radio (SDR) and its relatively newer version cognitive radio (CR). SDR, which has been introduced in [1], achieves significant improvements over services offered by current wireless networks. With SDR, the software embedded in a radio cell phone, for example, would define the parameters under which the phone should operate in real-time as its user moves from place to place. CR is even smarter than SDR. $\mathrm{CR}$ is designed to be aware of and sensitive to the changes in its surroundings. Thus it learns from its environment and performs functions that best serve its user. This is a very crucial feature of $\mathrm{CR}$ networks since, currently, the Federal Communications Commission (FCC) is reviewing its policies regarding the usage of licensed bands by unlicensed users [2]. In this context, the greatest advantage of $\mathrm{CR}$ is that it can be operated in licensed bands without a license.

Since the cognitive (unlicensed) users are utilizing the licensed band, they must detect the presence of licensed (primary) users in a very short time and must vacate the band for the primary users. Thus one of the major challenges that confronts this technology is: how do the cognitive (unlicensed) radios sense the presence of the primary (licensed) user? One may expect this to be trivial but as shown in [3], there are fundamental limits to the detection capabilities of $\mathrm{CR}$ networks. In this paper, we show improvement in spectrum sensing capabilities through cooperation between individual cognitive users.

Cooperative schemes with orthogonal transmission in a TDMA system have been recently proposed in [4] and [5].

$\dagger$ Corresponding Author: email : guru@ece.gatech.edu, phone/fax : 404 385-3012/894-7883
It has been shown in [4] that two user single hop networks in which one of the user acts as a relay for the other, result in lower outage probabilities. In particular, it is shown that the amplify-and-forward (AF) protocol [4], in which the relay transmits the signal obtained from the transmitter without any processing, achieves full diversity. In this paper, we study the effect of the AF cooperation protocol on the spectrum sensing capabilities of multi-user single-carrier cognitive radio networks. Analysis of multi-user single carrier networks can be found in [6]. In [6], we describe a cooperation scheme where the relay user is assumed to have no power constraint. However in practice all relay users have power constraint. In this paper, we describe a power constrained cooperation scheme.

The paper is organized as follows: In Section II, we describe the system model and formulate the problem of the primary user detection in a simple two user cooperative network. We also propose a practical cooperation scheme to detect the primary user. To show that cooperation leads to reduced detection time, in Section III, we consider a simple two-user asymmetric cognitive radio network and derive expressions for agility gain. In Section IV, we analyze multiuser single-carrier cognitive networks with and without centralized scheduling and derive precise conditions under which agility gain is achieved. Finally, in Section V, we present our conclusion.

We first introduce a few notations we shall be using throughout the paper. Consider any two non-negative functions functions $f(x)$ and $g(x)$. The following are standard notations [7]: 1) We say $f(x)=\Omega(g(x))$, if there exists a constant $C_{2}>0$ such that $f(x)>C_{2} g(x)$ for sufficiently large $x$.

2) We say $f(x)=\Theta(g(x))$ if there exists constants $C_{3}, C_{4}>$ 0 such that $C_{3} g(x) \leq f(x) \leq C_{4} g(x)$ for sufficiently large $x$.

The following are the symbols used throughout the paper:

- $U_{1}, U_{2}$ : Cognitive (unlicensed) users

- $P_{1}$ : Received power at $U_{1}$ due to the primary user

- $P_{2}$ : Received power at $U_{2}$ due to the primary user

- $G_{12}$ : Channel gain between $U_{1}$ and $U_{2}$

- $\theta$ : Primary user indicator; $\theta=1$ implies presence of the primary user and $\theta=0$ implies its absence

\section{COOPERATION SCHEME}

In this section, we describe the channel model that will be used throughout the paper, formulate the primary user detection problem and propose a practical cooperation scheme to improve the agility. 


\section{A. System Model}

In this paper, we assume that all channels experience Rayleigh fading. Moreover, channels corresponding to different cognitive users are assumed to be independent. If a signal $x$ is sent, the received signal $y$ is given by

$$
y=f x+w,
$$

where the fading coefficient $f$ and the additive noise $w$ are modelled as independent complex Gaussian random variables. Unless otherwise mentioned, the noise in this paper is assumed to be of zero mean and unit variance. We also assume that each user has access to its channel state information. This is facilitated by allowing the base station to transmit pilot symbols at regular intervals.

\section{B. Detection Problem}

An important requirement of a cognitive radio architecture is to detect the presence of primary or licensed users as quickly as possible. For this reason cognitive users should continuously sense the spectrum. Consider a network with two cognitive radio users $U_{1}$ and $U_{2}$ operating in a fixed TDMA mode for sending data to some base station. Suppose that a primary (licensed) user starts using the band. Then the two cognitive users need to vacate the band as soon as possible to make way for the primary user. However, the detection time becomes significant if one of the users, say $U_{1}$ is in the boundary of decodability as illustrated in Figure 1. The signal received from the primary user is so weak that the cognitive user $U_{1}$ takes a long time to sense its presence. We show that cooperation between the cognitive users can reduce the detection time of the "weaker" user thereby improving the "agility" of the overall network. We shall define these terms more precisely in Section III.

Throughout the paper, we allow the cognitive users, $U_{1}$ and $U_{2}$, to cooperate, with $U_{2}$ acting as a relay for the $U_{1}$. Figure 1 describes a scenario where two cognitive users $U_{1}$ and $U_{2}$ are engaged in transmitting data to a common receiver in a particular frequency band. Slotted transmission is used wherein $U_{1}$ and $U_{2}$ transmit in successive slots following the AF protocol [4] as shown in Figure 2. Accordingly in time slot $T_{1}, U_{1}$ transmits and $U_{2}$ listens. In time slot $T_{2}, U_{2}$ relays the information of the previous slot. Unknown to both these users, there is a primary user who has higher priority in occupying the band. It is crucial that presence of this primary user be detected as soon as possible. In time slot $T_{1}$, the signal received by $U_{2}$ from $U_{1}$ is given by,

$$
\tilde{y}=\theta h_{p 2}+a h_{12}+\tilde{w},
$$

where $h_{p i}$ denotes the instantaneous channel gain between the primary user and $U_{i}, h_{12}$ denotes the instantaneous channel gain between $U_{1}$ and $U_{2}$, and $\tilde{w}$ is the additive Gaussian noise. We assume that $h_{p 2}, h_{12}$, and $w_{2}$ are zero-mean complex Gaussian random variables which are pairwise independent. Also we assume that the channels are reciprocal, i.e., $h_{12}=$ $h_{21}$. In (1), a denotes the signal sent from $U_{1}$ such that $E\{a\}=0$ and $\theta$ denotes the primary indicator; $\theta=1$ implies presence of the primary user and $\theta=0$ implies its absence. If the transmit power constraint of $U_{1}$ is $P$ then,

$$
E\left\{\left|a h_{12}\right|^{2}\right\}=P G_{12},
$$

where $G_{12}=E\left\{\left|h_{12}\right|^{2}\right\}$ refers to the channel gain between the users $U_{1}$ and $U_{2}$. Since $h_{p 2}, h_{12}$ and $\tilde{w}$ are assumed pairwise independent, we have from (1) that

$$
E\left\{|\tilde{y}|^{2}\right\}=\theta^{2} P_{2}+P G_{12}+1 .
$$

where $P_{2}=E\left\{\left|h_{p 2}\right|^{2}\right\}$ refers to the received power at $U_{2}$ due to the primary user. In time slot $T_{2}$, the relay user $U_{2}$ relays the message from $U_{1}$ to a common cognitive receiver. The relay user has a maximum power constraint $\tilde{P}$. Hence it measures the average received signal power [8] and scales it appropriately so that its power constraint $\tilde{P}$ is satisfied. In slot $T_{2}$, when $U_{2}$ is relaying the message of $U_{1}$, we allow $U_{1}$ also to listen to its own message. Then the signal received by $U_{1}$ from $U_{2}$ is given by

$$
\begin{aligned}
y & =\sqrt{\beta} \tilde{y} h_{12}+\theta h_{p 1}+w \\
& =\sqrt{\beta} h_{12}\left(\theta h_{p 2}+a h_{12}+\tilde{w}\right)+\theta h_{p 1}+w,
\end{aligned}
$$

where $h_{p 1}$ is the instantaneous channel gain between the primary user and $U_{1}, w$ is additive Gaussian noise and $\beta$ is the scaling factor [4] used by $U_{2}$ to relay the information to the common receiver. In fact $\beta$ is given by [4] [8]

$$
\beta=\frac{\tilde{P}}{E\left\{|\tilde{y}|^{2}\right\}}=\frac{\tilde{P}}{\theta^{2} P_{2}+P G_{12}+1} .
$$

After the message component is cancelled, the user $U_{1}$ is left with the signal

$$
Y=\theta H+W,
$$

where $H=h_{p 1}+\sqrt{\beta} h_{12} h_{p 2}$ and $W=w+\sqrt{\beta} h_{12} \tilde{w}$. The detection problem can be now stated as:

Given the observation

$$
Y=\theta H+W
$$

the detector decides on

$$
\mathcal{H}_{1}: \theta=1
$$

or

$$
\mathcal{H}_{0}: \theta=0 \text {. }
$$

In [6], we describe a variant of the above algorithm where there is no power constraint on the relay user $U_{2}$. However in practice all users have power constraint. The detection problem for the constrained cooperation scheme described above is identical to the unconstrained cooperation scheme in [6] (see Eq.(3) in [6]). As in [6], we use the energy detector (ED) [9] to detect the presence of primary user. Since the derivation of the detection probability is very similar to the unconstrained cooperation scheme in [6], we skip the details in this paper and present the final result. The details can be found in [6]. Defining

$$
\varphi(t ; a, b)=\int_{0}^{\infty} e^{-h-\frac{t}{a+b h}} d h
$$


for positive $t, a$, and $b$, we have the following proposition: Proposition 1: Let $p_{c}^{(1)}$ and $p_{n}^{(1)}$ denote the detection probabilities of $U_{1}$ with and without cooperation from $U_{2}$ respectively. We have

$$
p_{c}^{(1)}=\varphi\left(\lambda ; P_{1}+1, \tilde{\beta}\left(P_{2}+1\right)\right)
$$

and

$$
p_{n}^{(1)}=\alpha^{\frac{1}{P_{1}+1}}
$$

where

$$
\tilde{\beta}=\frac{\tilde{P} G_{12}}{\theta^{2} P_{2}+P G_{12}+1}
$$

and $\lambda$ is uniquely determined by

$$
\varphi\left(\lambda ; 1, \frac{\tilde{P} G_{12}}{P G_{12}+1}\right)=\alpha .
$$

In Figure 3 , we have plotted $p_{n}^{(1)}$ and $p_{c}^{(1)}$ as a function of $P_{2}$ for $P=\tilde{P}=1$. For a path-loss exponent of 3.5 , we have plotted $p_{n}^{(1)}$ and $p_{c}^{(1)}$ for three different values of $P_{1}: P_{1}=1,2.5$, and 6 . For each value of $P_{1}$, we note that the constrained cooperation scheme is beneficial $\left(p_{c}^{(1)}>p_{n}^{(1)}\right)$ only for a certain range of the power level at $U_{2}, P_{2}$. Also the maximum achievable probability gain is dependent on the position of the cognitive user $U_{1}$.

From Figure 3, we see that the maximum gain is achieved when $U_{2}$ is optimally placed between the primary user and $U_{1}$. When $P=\tilde{P}=1$, we have extensively evaluated (2) and (3) for various values of false alarm probability $\alpha$ and path loss exponent $\delta$. Using numerical calculation, we can easily confirm the following fact:

Observation 1: If $P_{2}=G_{12}$, then

$$
p_{c}^{(1)}>p_{n}^{(1)} \text {. }
$$

This is supported by Figure 4 where we have plotted $p_{c}^{(1)}-p_{n}^{(1)}$ as a function of $P_{1}$ for different values of $\delta$ between 3 and 6. This Observation is useful in Section IV, when we discuss optimal pairing schemes for random networks.

\section{AgILITy OF THE TWO USER COGNITIVE RADIO NETWORK}

So far, we have devoted ourselves to improvement in detection probabilities through cooperation. The final goal, however, is to reduce the overall detection time. To show the effect of cooperation on the overall detection time, we shall define two types of protocols employing different degrees of cooperation. We assume that there is a common base station (capable of both receiving and sending) with which all the cognitive users communicate.

1) Non Cooperative (NC) Protocol: All the users detect the primary user independently. However the first user to detect the presence of the primary user informs the other users through the common receiver.

2) Totally Cooperative (TC) Protocol: This employs the constrained cooperation scheme described in Section II. Thus two users operating in the same carrier, if placed sufficiently near to each other, cooperate to find the presence of the primary user. The first user to detect the presence of the primary user informs the others through the common base station.

Let $T_{n}$ and $T_{t}$ denote the average time taken by the two user network described in Section II to detect the primary user under NC and TC protocol, respectively. As before, let $p_{c}^{(1)}$ and $p_{n}^{(1)}$ be given by (2) and (3). We have the following proposition.

Proposition 2: If $p_{n}^{(2)}=\alpha^{\frac{1}{P_{2}+1}}$, then we have,

$$
T_{n}=\frac{2-\frac{p_{n}^{(1)}+p_{n}^{(2)}}{2}}{p_{n}^{(1)}+p_{n}^{(2)}-p_{n}^{(1)} p_{n}^{(2)}},
$$

and

$$
T_{t}=\frac{2-\frac{p_{c}^{(1)}+p_{n}^{(2)}}{2}}{p_{c}^{(1)}+p_{n}^{(2)}-p_{c}^{(1)} p_{n}^{(2)}} .
$$

We define the agility gain of the TC protocol over the NC protocol as

$$
\mu_{n / t} \triangleq \frac{T_{n}}{T_{t}}
$$

In Figure 5, we have plotted the agility gain $\mu_{n / t}$ under the constrained cooperation scheme. We find that as $U_{1}$ is farther from the primary user, the maximum agility gain increases. When $P_{1}=1$, the maximum agility gain is seen to be approximately 1.5 . This implies a decrease in the detection time by approximately $34 \%$. Since the cognitive users need to continuously monitor spectrum, a decrease of $34 \%$ in the detection time is very useful in the long term.

\section{Multiuser Cognitive network}

Throughout the section, we have considered the simple case of two user network. What happens when there are more than two users? How can we cooperate the users to achieve agility gain? In this section, we shall consider the presence of more than two cognitive users. Ideally since there are many users, we would like to find an optimal cooperation algorithm (possibly) involving more than two users that would achieve even more gain than a simple two-user network. However, this leads to complex detection algorithms which perform better only at the cost of great computational complexity. We know from Sections II and III that grouping the users into pairs leads to increased detection probability if in each pair, one user acts as a relay for the other. Thus an alternative solution would be to find a relay for each user who seeks help and to hope that the overall detection time is reduced. Can we find a relay user for each user who needs help? Can we still achieve agility gain over non-cooperation?

Let $\left\{U_{k}\right\}_{k=1}^{m}$ denote the set of users inside the boundary of decodability of the primary user. Further let $P_{k}$ denote the received power from the primary user at cognitive user $U_{k}$. If $P_{\text {low }}$ denotes the received power due to the primary user at the boundary of decodability, then all the cognitive users with power level $P_{k}>P_{\text {low }}$ have to be vacated as soon as possible for the primary user. Since the cognitive users cannot be arbitrarily close to the primary user, we assume $P_{k}<P_{u p p}$ for some finite $P_{u p p}$. For any two power levels $P_{A}$ and $P_{B}$, define

$$
\mathcal{U}\left(P_{A}, P_{B}\right)=\left\{U_{k}: P_{A} \leq P_{k} \leq P_{B}\right\}
$$


In the constrained cooperation scheme of Section II, we find that for a particular cognitive user $U_{1}$ to be benefitted by cooperation, it is essential that the relay user $U_{2}$ is closer to the primary user than $U_{1}$ (see Figure 3 ). In general, it is practically difficult to assign a relay user for each cognitive user. In such a case, we define the critical power $P^{*}$ of a detection scheme in the following way: If a particular cognitive user $U_{k} \in \mathcal{U}\left(P_{\text {low }}, P^{*}\right)$, we allow the user $U_{k}$ to search for a relay user. If on the other hand $U_{k} \in \mathcal{U}\left(P^{*}, P_{\text {upp }}\right)$ then $U_{k}$ does not seek help from anyone. The rationale behind this is that users close to the primary user already have good detection probabilities. So they can help the users far away from the primary user to improve their detection probabilities. We can visualize the above procedure the following way: Any cognitive user $U_{k} \in \mathcal{U}\left(P_{\text {low }}, P_{\text {upp }}\right)$ causes interference to the primary user and must be vacated. We have divided the user set $\mathcal{U}\left(P_{\text {low }}, P_{\text {upp }}\right)$ into two subsets $\mathcal{A}=\mathcal{U}\left(P_{\text {low }}, P^{*}\right)$ and $\mathcal{B}=\mathcal{U}\left(P^{*}, P_{\text {upp }}\right)$ such that users in $\mathcal{A}$ seek help from users in $\mathcal{B}$. Let $n$ and $\tilde{n}$ be the number of users in $\mathcal{A}$ and $\mathcal{B}$ respectively and let $m=n+\tilde{n}$.

In general, there can be many ways to determine the critical power, $P^{*}$. For example, we can fix a threshold probability of detection $p^{*}$ such that if the detection probability of a cognitive user is greater than $p^{*}$ without cooperation then it does not search for any relay user. From (3), we find that $P^{*}$ is uniquely determined by

$$
\alpha^{\frac{1}{P^{*}+1}}=p^{*}
$$

yielding

$$
P^{*}=\left(\frac{\ln \left(\alpha / p^{*}\right)}{\ln p^{*}}\right)
$$

where $\alpha$ is the tolerable false alarm probability. Throughout this paper we discuss only single-carrier multi-user networks. Discussion of multi-carrier multi-user networks can be found in [6].

\section{A. Centralized Networks}

In this section, we shall assume that all the $m=n+\tilde{n}$ users are in $\mathcal{U}\left(P_{\text {low }}, P_{\text {upp }}\right)$ and operate in the same frequency band. Consider two users $U_{1} \in \mathcal{U}\left(P_{\text {low }}, P^{*}\right)$ and $U_{2} \in \mathcal{U}\left(P^{*}, P_{\text {upp }}\right)$ with $G_{12}$ being the channel gain between them. Further let $U_{2}$ act as a relay for $U_{1}$ and let the position of $U_{1}$ be fixed, i.e., let $P_{1}$ be fixed. We search through the $\left(P_{2}, G_{12}\right)$ space to find an optimal relay user for $U_{1}$. As before, let $p_{c}^{(1)}$ and $p_{n}^{(1)}$ given by (2) and (3) denote the detection probabilities of $U_{1}$ with and without cooperation from $U_{2}$, respectively. Define

$$
\mathcal{P}\left(P_{1}\right)=\left\{\left(P_{2}, G_{12}\right): p_{c}^{(1)}>p_{n}^{(1)}\right\}
$$

to be the critical region for the user $U_{1}$. It is obvious that $U_{1}$ can benefit from cooperation only if the relay user $U_{2}$ is inside its critical region $\mathcal{P}\left(P_{1}\right)$.

Without loss of generality, we let $\mathcal{U}\left(P_{\text {low }}, P^{*}\right)=\left\{U_{k}\right\}_{k=1}^{n}$ and $\mathcal{U}\left(P^{*}, P_{\text {upp }}\right)=\left\{U_{k}\right\}_{k=n+1}^{m}$. In this section, we assume that there is a centralized scheduler that implements the cooperation protocol described below.

If all the users in $\mathcal{U}\left(P_{\text {low }}, P_{\text {upp }}\right)$ follow the cooperation protocol, we say that the users follow the TC protocol. If

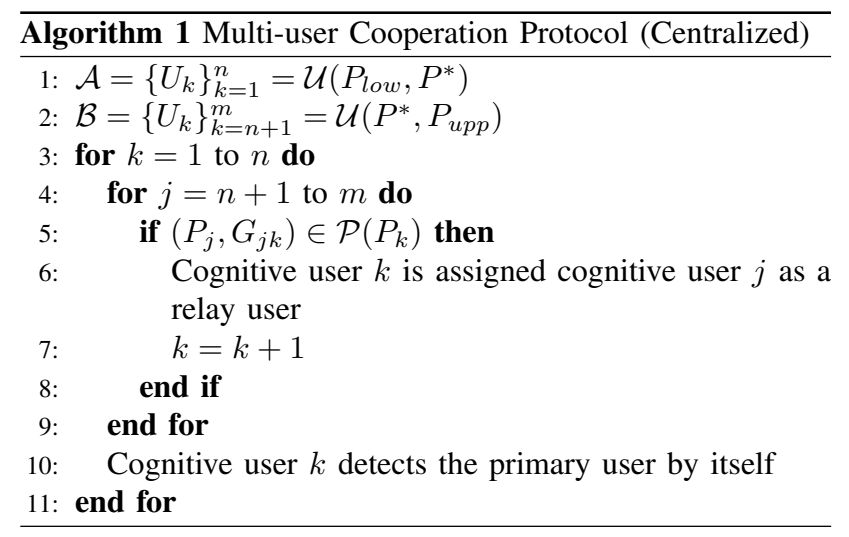

on the other hand, all the users detect the primary user by themselves with no cooperation, they are said to follow the NC protocol.

Since all users are operating in a single carrier, we allow them to operate in a TDMA mode. In that case, the order in which the users operate becomes an important issue. Let $p_{k}$ denote the detection probability of the $k^{t h}$ cognitive user. Suppose now that the detection probabilities satisfy $p_{1} \leq p_{2} \leq$ $\ldots . \leq p_{m}$ and we follow the ordering $(m, m-1, \ldots, 1)$. Then the detection time is the least among all permutations of the users since the cognitive user with the best chance of detecting the primary user operates first. However this is always not guaranteed. In general, following a suboptimal ordering can result in more time for detection. When there are $m$ users there are $m$ ! ways to arrange their operation cycle. Any ordering $\mathcal{S}$ is an element of $\mathcal{T}_{m}$.

Given an ordering $\mathcal{S} \in \mathcal{T}_{m}$, let $T_{n}(m \mid \mathcal{S})$ and $T_{t}(m \mid \mathcal{S})$ denote the time taken to detect the primary user when all the $m$ users are under NC protocol and TC protocol respectively. We have the following result:

Proposition 4: Let $m$ be the number of cognitive users operating in a single carrier. Then for any given ordering $\mathcal{S} \in \mathcal{T}_{m}$, we have

$$
1<T_{t}(m \mid \mathcal{S}) \leq T_{n}(m \mid \mathcal{S})
$$

However, as argued before, there is a possibility that the users with the best chance of finding the primary user operate after a long time in a cycle. To improve the detection capability, we allow users to operate in a time-hopping fashion. Hence after every cycle involving the operation of all the $m$ users, we choose an ordering randomly from $\mathcal{T}_{m}$. Note that in this section, we assume that there exists a centralized scheduler to pair the users. Thus one can argue that the scheduler can select the optimal ordering $\mathcal{S}_{\text {opt }} \in \mathcal{T}_{m}$ where

$$
\mathcal{S}_{\text {opt }}=\underset{\mathcal{S} \in \mathcal{T}_{m}}{\arg \min }\left\{T_{t}(m \mid \mathcal{S})\right\}
$$

However our ultimate goal is to develop decentralized cooperation schemes. With this in mind, we adopt the random ordering strategy.

Since all the orderings are equally likely, we get that the average detection time in case of $\mathrm{TC}$ and $\mathrm{NC}$ protocol are 
given, respectively, by

$$
T_{t}(m)=\frac{1}{m !} \sum_{\mathcal{S} \in \mathcal{T}_{m}} T_{t}(m \mid \mathcal{S})
$$

and

$$
T_{n}(m)=\frac{1}{m !} \sum_{\mathcal{S} \in \mathcal{T}_{m}} T_{n}(m \mid \mathcal{S}) .
$$

We define the agility gain in this case to be

$$
\mu_{m}=\frac{T_{n}(m)}{T_{t}(m)}
$$

and also let

$$
\mu_{\infty}=\lim _{m \rightarrow \infty} \mu_{m}
$$

if it exists. The following result summarizes the behaviour of asymptotic agility gain.

Proposition 5: Let $m$ be the total number of users operating in a single carrier. Then

(a) For any $m$, we have,

$$
1 \leq \mu_{m}<\infty
$$

(b) When $\mu_{m} \rightarrow \mu_{\infty}$, we have

$$
1 \leq \mu_{\infty}<\infty
$$

Since there is a possibility of $\mu_{\infty}=1$, we wish to know when there is agility gain. Consider two users $U_{1} \in \mathcal{U}\left(P_{\text {low }}, P^{*}\right)$ and $U_{2} \in \mathcal{U}\left(P^{*}, P_{\text {upp }}\right)$ with $U_{2}$ acting as a relay for $U_{1}$. As before, let $p_{c}^{(1)}$ and $p_{n}^{(1)}$ given by (2) and (3) denote the detection probabilities of $U_{1}$ with and without cooperation from $U_{2}$ respectively.

Definition: We say that cognitive user $U_{1} \in \mathcal{U}\left(P_{\text {low }}, P^{*}\right)$ is a happy user if

$$
p_{c}^{(1)}-p_{n}^{(1)} \geq \epsilon_{0}
$$

for some $\epsilon_{0}>0$ and independent of $P_{1}, P_{2}$ and $G_{12}$. Let $c(n)$ denote the number of happy users out of the $n$ users in $\mathcal{U}\left(P_{\text {low }}, P^{*}\right)$. The following theorem gives a simple sufficient condition for the agility gain to be greater than one.

Theorem 1:(Agility Gain Theorem) Let $n$ and $\tilde{n}$ denote the number of users in $\mathcal{U}\left(P_{\text {low }}, P^{*}\right)$ and $\mathcal{U}\left(P^{*}, P_{\text {upp }}\right)$ respectively. Then as $m=n+\tilde{n} \rightarrow \infty$, we have

(a) If $\frac{n}{\tilde{n}} \rightarrow 0$, then $\mu_{m} \rightarrow 1$.

(b) If $n=\Omega(\tilde{n})$ and $c(n)=\Omega(n)$ then

$$
\mu_{m}=1+\Omega(1) .
$$

Since $c(n) \leq n$, the previous theorem implies that we get agility gain from cooperation if $c(n)=\Theta(n)$. The worst case occurs when all users in $\mathcal{U}\left(P_{\text {low }}, P^{*}\right)$ are scheduled to transmit first. Though the primary user will eventually be detected, the time taken will be greatly reduced in case of cooperative networks since each user in $\mathcal{U}\left(P_{\text {low }}, P^{*}\right)$ is linked with a user in $\mathcal{U}\left(P^{*}, P_{\text {upp }}\right)$ whose help can greatly improve the detection probability and hence the overall agility.

\section{B. Decentralized Networks}

The results of Section IV.A can be used to design practical pairing schemes when the location of users are random and there is no centralized scheduler. Let us assume that the location of the primary user is known to all the cognitive users. Thus each user knows its position from the primary user. We assume that the power level $P_{k}$ of the cognitive user $U_{k}$ is uniformly distributed in $\left[P_{l o w}, P_{u p p}\right]$. Let a particular cognitive user $U_{1}$ be in $\mathcal{U}\left(P_{\text {low }}, P^{*}\right)$. In the previous section, we assumed that there was a centralized scheduler which assigned a relay user for $U_{1}$ inside its critical region $\mathcal{P}\left(P_{1}\right)$. However now there is no centralized scheduler and in general $\mathcal{P}\left(P_{1}\right)$ may not be even of regular shape. Also all the cognitive users have limited transmission range. Thus $G_{12} \geq G_{\text {low }}$ for some positive $G_{l o w}$. Since the distance between any two cognitive users is strictly positive, $G_{12} \leq G_{u p p}$ for some finite $G_{\text {upp }}$. In such a practical situation, how does the user $U_{1}$ find a relay such that (4) is satisfied. An ad-hoc solution would be the following:

(1) For some integer $N$, partition $\left[P_{l o w}, P^{*}\right]$ into $N$ subintervals $\left\{\mathcal{I}_{l}\right\}_{l=1}^{N}$ where each $\mathcal{I}_{l}$ is of width $\frac{P^{*}-P_{l o w}}{N}$

(2) Partition $\left[P^{*}, P_{u p p}\right]$ into $N$ subintervals $\left\{\mathcal{J}_{l}\right\}_{l=1}^{N}$ where each $\mathcal{J}_{l}$ is of width $\frac{P_{u p p}-P^{*}}{N}$.

(3) Partition $\left[G_{l o w}, G_{\text {upp }}\right]$ into $N$ subintervals $\left\{\mathcal{G}_{l}\right\}_{l=1}^{N}$ where each $\mathcal{G}_{l}$ is of width $\frac{G_{u p p}-G_{\text {low }}}{N}$.

(4) For each $\mathcal{I}_{l}$ determine a region $\mathcal{J}_{l}$ and $\mathcal{G}_{l}$ with the following property: If a cognitive user $U_{1}$ is in $\mathcal{I}_{l}$ and is able to find a relay user $U_{2}$ in $\mathcal{J}_{l}$ and $G_{12} \in \mathcal{G}_{l}$, then $U_{1}$ is a happy user, i.e., (4) is satisfied.

(5) Assign label $l$ to users in $\mathcal{I}_{l}$ and $\mathcal{J}_{l}$.

Thus in effect we have discretized the search space for each cognitive user. This allows practical implementation. Also for a user $U_{k}$ in $\mathcal{U}\left(P_{\text {low }}, P_{\text {upp }}\right)$ let $l(k)$ denote its label. We say that a cooperative detection scheme has a pairing protocol if we are able to do steps (1)-(5) mentioned above. We now ask the question: Does there exist a pairing protocol for our cooperation scheme? The following theorem answers the question:

Theorem 2: Suppose Observation 1 holds, i.e., for any two users $U_{1}$ and $U_{2}$, if $P_{2}=G_{12}$ then $p_{c}^{(1)}>p_{n}^{(1)}$. Then the constrained cooperation scheme of Section II has a pairing protocol.

We describe the decentralized pairing protocol below:

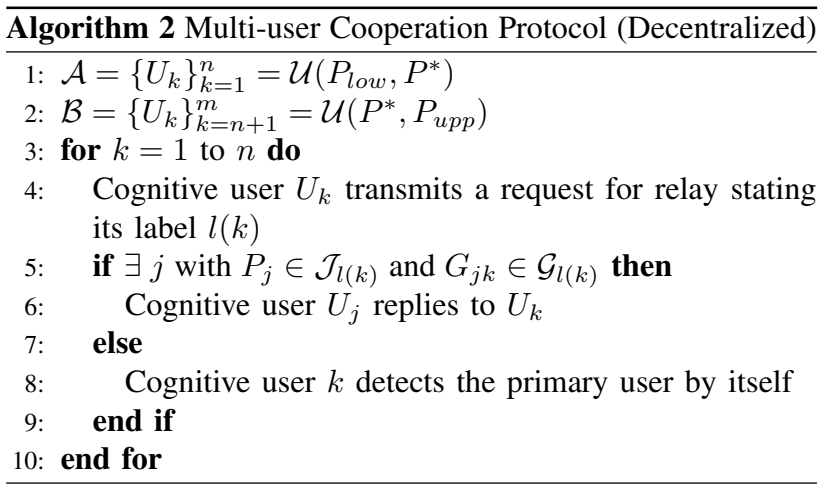


In step (5) of the above protocol, if more than one user responds back to a cognitive user $U_{1}$ seeking help, then $U_{1}$ chooses one of the users randomly. As before, let $\tilde{n}$ denote the number of users in $\mathcal{U}\left(P^{*}, P_{\text {upp }}\right)$ and $n=m-\tilde{n}$ denote the number of users in $\mathcal{U}\left(P_{\text {low }}, P^{*}\right)$. We know from the Agility Gain theorem, that when $\frac{n}{\tilde{n}} \rightarrow 0$ there is no agility gain. Let us then assume that $n=\Omega(\tilde{n})$ and let $c(n)$ be the number of happy users in $\mathcal{U}\left(P_{\text {low }}, P^{*}\right)$. If we can ensure that

$$
c(n)=\Omega(n),
$$

our scheme is definitely beneficial. Can we ensure (5) with probability 1 ? The following theorem tells when that is possible.

Theorem 3: Under the hypothesis of Theorem 2, as $m \rightarrow \infty$,

$$
\frac{c(n)}{n}=1
$$

with probability 1 if and only if $\tilde{n} \rightarrow \infty$.

Thus we have ensured that $\mu_{m}=1+\Omega(1)$ with probability 1. In essence, we have developed a practical algorithm for pairing in random networks which is asymptotically optimal and thus has reduced detection time. The algorithm is very important in practical systems because the cognitive users need to continuously sense the spectrum and shift bands to avoid interference with the primary users. In this context, algorithms which reduce detection time even by a small amount are very useful.

\section{CONCLUSION AND FUTURE WORK}

In this paper, we have shown the benefits of cooperation in increasing the agility of cognitive radio systems. We have taken an asymmetric two user cooperative cognitive network and shown improvement in probability of detection leading to enhanced agility. We have then considered the general $n$ user single carrier cognitive network and derived a simple sufficient condition for asymptotic agility gain. We have also considered the case when the users are randomly distributed and stated conditions under which agility gain can be achieved.

In this paper, we have assumed the location of the primary transmitter. In future, we wish to develop cooperative protocols when the primary transmitter location is unknown to the cognitive users. Also, we wish to consider other practical issues like mobility of the cognitive users, effect of shadowing and the presence of more than one cognitive cluster.

\section{REFERENCES}

[1] I. J. Mitola, "Software radios: Survey, critical evaluation and future directions," IEEE Aerosp. Electron. Syst. Mag., vol. 8, pp. 25-31, Apr. 1993.

[2] FCC, "Spectrum policy task force report," ET Docket No. 02-155, Nov. 2002.

[3] A. Sahai, N. Hoven and R. Tandra, "Some fundamental limits in cognitive radio," in Proc. Allerton Conf. on Commun., Control and Computing, Oct. 2004.

[4] J. N. Laneman and D. N. C. Tse, "Cooperative diversity in wireless networks: Efficient protocols and outage behaviour," IEEE Trans. Inform. Theory, vol. 50, pp. 3062-3080, Dec. 2004.

[5] J. N. Laneman and G. W. Wornell, "Distributed space-time coded protocols for exploiting cooperative diversity in wireless networks," IEEE Trans. Inform. Theory, vol. 49, pp. 2415-2425, Oct. 2003.

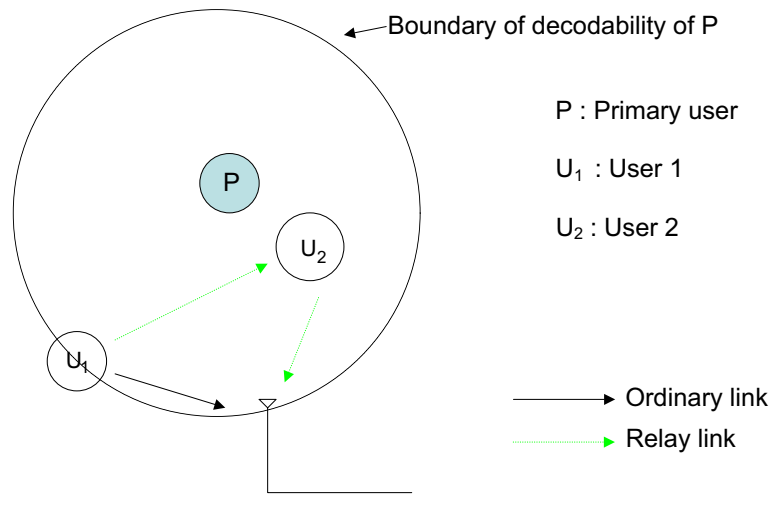

Fig. 1. Cooperation in cognitive network

$$
\begin{array}{|l|l|l|l|l|}
\hline \mathrm{U}_{1} \mathrm{Tx} & \mathrm{U}_{2} \text { Relay } & \mathrm{U}_{2} \mathrm{Tx} & \mathrm{U}_{1} \text { Relay } & \cdots \\
\hline
\end{array}
$$

Fig. 2. Relay protocol used

[6] G. Ganesan and Y. G. Li, "Agility improvement through cooperative diversity in cognitive radio networks," in GLOBECOM 2005, St. Louis, Missouri, accepted for publication.

[7] C. E. L. T. H. Cormen and R. L. Rivest, Introduction to Algorithms, 1st ed. MIT Press, 1990.

[8] I. Hammerstrom, M. Kuhn and A. Wittneben, "Cooperative diversity by relay phase rotations in block fading environment," in Fifth IEEE Workshop on Signal Process. Advances in Wireless Commun., July 2004.

[9] H. V. Poor, An Introduction to Signal Detection and Estimation, 2nd ed. Springer-Verlag, 1994. 


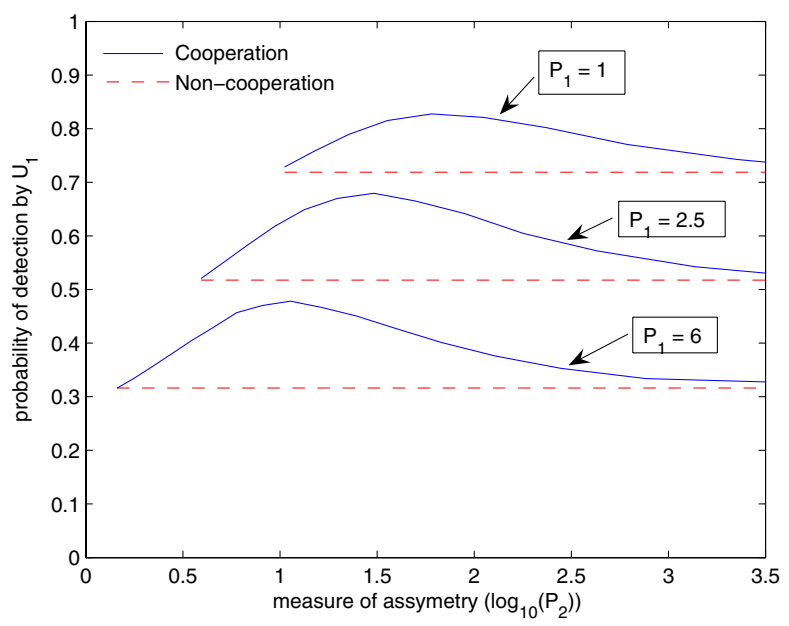

Fig. 3. Cooperation improves probability of detection

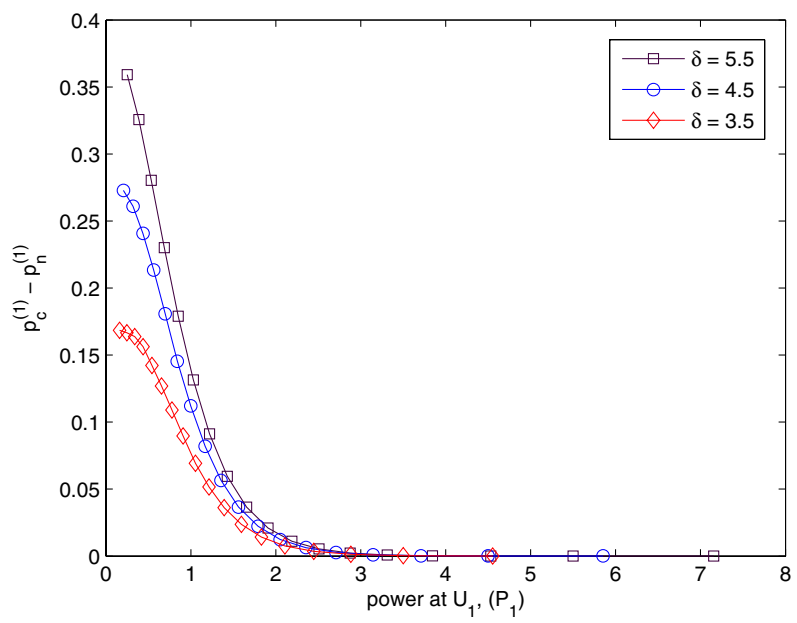

Fig. 4. Increase in detection probability when $U_{2}$ is placed optimally between $U_{1}$ and the primary user

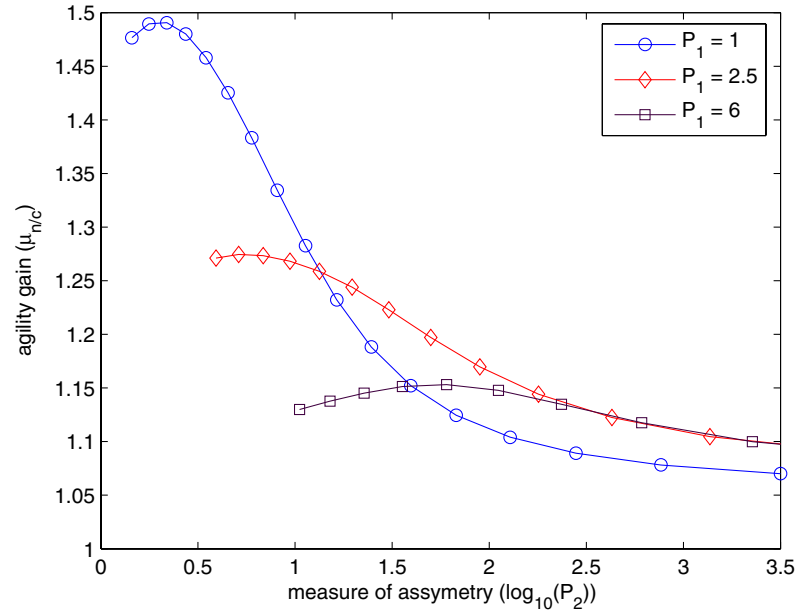

Fig. 5. Agility gain in two user networks under the constrained scheme 